\title{
Kinematic Analysis on Materials on Counter Flow Screen and Selection of Dynamic Parameters of Machine
}

\author{
Jing Jiang ${ }^{1, a}$, Qingkai Han ${ }^{1, b}$, Yuanhua Xie ${ }^{1, \mathrm{c}}$, Jin $\operatorname{Han}^{1, \mathrm{~d}}$ and Shuying Liu ${ }^{1, \mathrm{e}}$ \\ ${ }^{1}$ School of Mechanical Engineering and Automation, Northeastern University, \\ Shenyang 110004, P. R. China \\ ajjiang@mail.neu.edu.cn, bqhan@mail.neu.edu.cn, cyhxie@mail.neu.edu.cn, \\ djinhan@mail.neu.edu.cn, èshuyliu45@163.com
}

Keywords: Counter flow screen, Kinematic analysis, Dynamical parameters, Sorting mechanism.

\begin{abstract}
The paper presents the structural characteristics of counter flow screen. Pieces of plastic film and sandstones with different friction factors and elasticity coefficients are chosen as the research objects. The motion state and the sorting mechanism of materials in the sorting process are studied. According to the motion analysis on pieces of plastic film and sandstones on counter flow screen, it can be concluded that the materials with different friction factors and elasticity coefficients can all be sorted by counter flow screen. The more difference they have in friction factors and elasticity coefficients, the better sorting performance can be achieved. The selection and computation on the dynamical parameters of counter flow screen is carried out, which provides the theory evidence for the design of new friction sorting equipment and opens up new prospects for the application of counter flow screen.
\end{abstract}

\section{Introduction}

Most of the treatment with environmental pollution is passively executed in China. If there is no mandatory order from environmental protection agencies or government departments, few enterprises would like to implement this work actively. Especially with gradual increasing of individual operations, this situation is becoming worse and worse. Therefore, it is very important to develop energy-saving and environmental protection equipment with China's actual conditions, low investment, easy operation, good performance and low energy consumption [1-4].

Along with economic development and urbanization process, more and more municipal solid wastes cause increasingly prominent urban environmental problems and bring tremendous pressure to the natural environment. For a long period of time, people's lack of awareness on environmental pollution has resulted in the growing problems of urban pollution. Municipal solid wastes such as plastic bags and pieces of broken plastic film create so-called white pollution in many cities. Although a large number of new technologies have been developed for the harmless and resource processing on solid wastes, the capacity of treatment is relatively small and far below the amount of garbage generated. Counter flow screen originated from China is a kind of sorting equipment to separate solid wastes by friction [5-7]. In this paper, the motion state of materials with different friction factors and elasticity coefficients on counter flow screen is analyzed in order to apply the separation function of vibrating screen to dispose solid wastes including pieces of plastic film. It is of great significance to waste recycling, elimination of pollution hazards and environmental protection.

\section{Structural Characteristics of Counter Flow Screen}

Counter flow screen is made up of screen box, screen surface, vibration exciter, main vibration spring $k$, link spring $k_{0}$, guide rod, chassis and other parts, as shown in Fig.1. The motor runs to drive the eccentric shaft to rotate, so that flexible connecting rod drives the machine body to make the approximate straight line reciprocating motion in order to realize the sorting for solid wastes. 
The vibrating screen like this has several screen surfaces with different angles. In accordance with different materials, the angle of each screen can be accordingly adjusted. Besides, counter flow screen has other merits such as simpler structure, fewer parts, fewer maintenance points and easier maintenance. However, a large dynamic load is exerted on the base. In order to overcome this, spring shock absorber can be added between chassis and base.

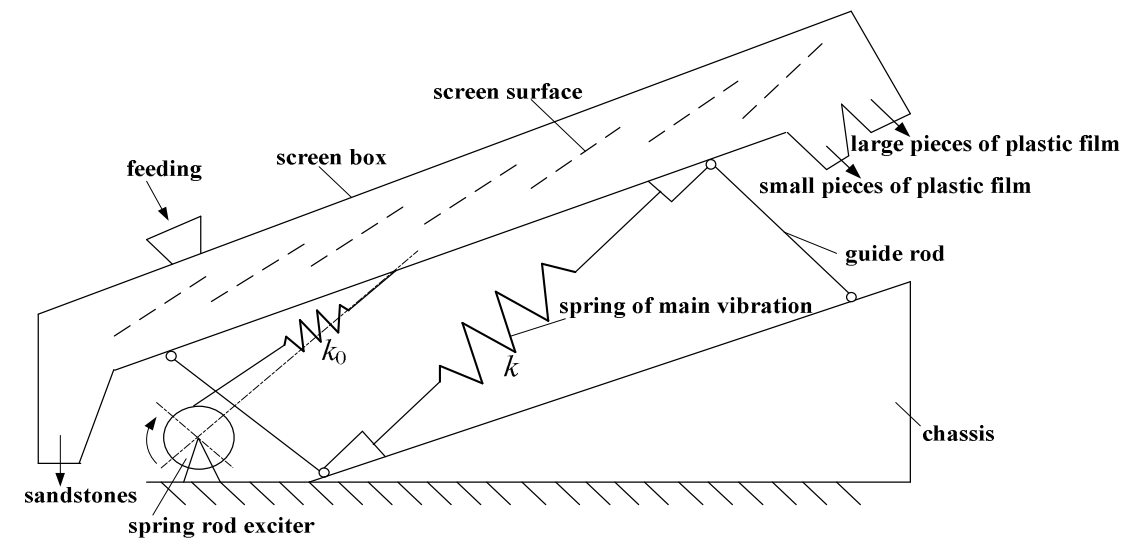

Fig. 1. Structure of counter flow screen

\section{Motion State of Materials in the Process of Sorting}

Counter flow screen is a novel screen to separate the solid wastes according to their different friction factors and elasticity coefficients. The motion state of materials on the screen surface is closely related to the movement of screen surface and the friction factors and elasticity coefficients between screen surface and materials. If the screen machine vibrates at different frequencies, the materials on screen surface will perform different motion states.

Forward movement of material particles (Sliding or rolling). When the movement of materials of thin layer on the screen surface is studied, the interaction among materials is nearly negligible; While if the layer of materials is very thick, the impact factor of the material layer thickness should be considered. This method is not only simple, but also practical.

The forces on the materials on the screen surface are shown as Fig.2. When the acceleration of screen surface is negative and the materials move along with the screen surface, inertia force plus gravity for the materials along screen surface equals $F_{+}$; the limiting friction between screen surface and materials is $F$, as shown in equation (1) and (2).

$$
\begin{aligned}
& F_{+}=-m \ddot{x}-G \sin \alpha \\
& F=f N=f(m \ddot{y}+G \cos \alpha)
\end{aligned}
$$

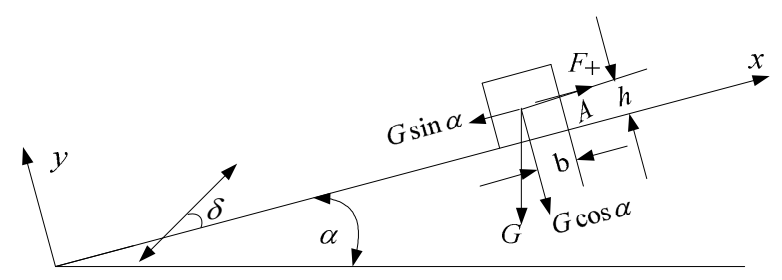

Fig. 2. Forces on the materials on the screen surface (I)

When $F_{+}$is not less than $F$ like equation (3), material particles will slide forward along the screen surface, that is, material particles will slide upward along the screen surface,

$$
-m \ddot{x}-G \sin \alpha \geq f(m \ddot{y}+G \cos \alpha)
$$

where, $m$ is the mass of material particles, $\mathrm{kg} ; G$ is the gravity of material particles, $\mathrm{N} ; \alpha$ is the inclined angle of screen surface, ${ }^{\circ} ; N$ is positive pressure material particles act on screen surface, $\mathrm{N}$; $f$ is the friction factor between material particles and screen surface, $f=\tan \mu$ ( $\mu$ is the static angle 
of sliding friction); $\ddot{x}, \ddot{y}$ are the acceleration for screen machine along the $\mathrm{x}$ and $\mathrm{y}$ direction, $\ddot{x}=-r \omega^{2} \sin \varphi \cos \delta, \ddot{y}=-r \omega^{2} \sin \varphi \sin \delta ; \quad r$ is the distance from the centroid of eccentric block to the rotation axis; $\omega$ is the rotation angular velocity of axis; $\varphi$ is the vibration phase angle; $\delta$ is the vibration direction angle. When the torque at point $\mathrm{A}$ is $F_{+} h>N b$, material particles appear to roll forward, as shown in equation (4).

$$
F_{+} \geq \frac{b}{h} N
$$

If $f<b / h$, material particles appear sliding; If $f>b / h$, material particles appear rolling. For flat granules of materials, the opportunities of sliding are more than that of rolling; for spherical particles of materials, the opportunities of rolling are more than that of sliding; In the sorting process of solid wastes, there is rarely rolling, so only sliding state is analyzed here. Expression $\ddot{x}$ and expression $\ddot{y}$ are substituted into equation (3) and simplified to get equation (5). When $\sin \varphi_{+}=1$, minimum inertial acceleration $a_{1}$ which makes materials slide forward can be calculated as equation (6), and minimum rotating speed of screen $n_{+\min }$ which makes materials move forward can be calculated as equation (7). Where, $g$ is acceleration of gravity, $\mathrm{m} / \mathrm{s}^{2}$; In order to produce sliding forward of materials on the screen surface, the working speed of screen should be $n>n_{+\min }$.

$$
\begin{aligned}
& r \omega^{2} \sin \varphi_{+} \geq \frac{g \sin (\mu+\alpha)}{\cos (\mu-\delta)} \\
& a_{1} \geq \frac{g \sin (\alpha+\mu)}{\cos (\mu-\delta)} \\
& n_{+\min } \geq 30 \sqrt{\frac{g \sin (\mu+\alpha)}{r \pi^{2} \cos (\mu-\delta)}} \quad(\mathrm{r} / \mathrm{min})
\end{aligned}
$$

Backward movement of material particles (Sliding or rolling). As shown in Fig.3, when the acceleration of screen surface is positive and the materials move along with the screen surface, not only a positive slide is generated, but also the reverse slide. At the moment of the beginning of reverse sliding, $x$-direction component of inertia force plus $x$-direction component of gravity is $F_{-}$in equation (8). If $F_{-} \geq F$, materials appear the reverse sliding along screen surface, that is, along the $\mathrm{x}$-direction reverse sliding down as shown in equation (9).

$$
\begin{aligned}
& F_{-}=m \ddot{x}+G \sin \alpha \\
& m \ddot{x}+G \sin \alpha \geq f(m \ddot{y}+G \cos \alpha)
\end{aligned}
$$

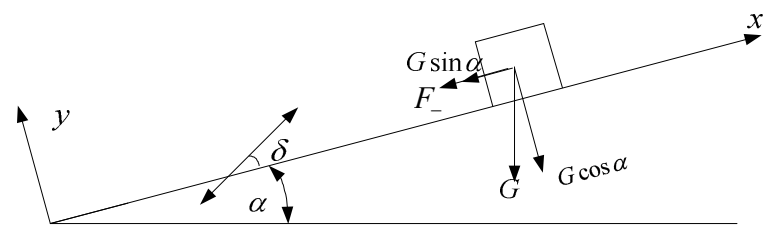

Fig. 3. Forces on the materials on the screen surface (II)

Expression $\ddot{x}$, expression $\ddot{y}$ and $f=\tan \mu$ are substituted into equation (9) and simplified to get equation (10). When $\sin \varphi_{-}=-1$, minimum inertial acceleration $a_{2}$ which makes materials slide backward can be calculated as equation (11), and minimum rotating speed of screen $n_{-\min }$ which makes materials move backward can be calculated as equation (12). In order to produce sliding backward of materials on the screen surface, the working speed of screen should be $n>n_{-\min }$. 


$$
\begin{aligned}
& -r \omega^{2} \sin \varphi_{-} \geq \frac{g \sin (\mu-\alpha)}{\cos (\mu+\delta)} \\
& a_{2} \geq \frac{g \sin (\mu-\alpha)}{\cos (\mu+\delta)} \\
& n_{- \text {min }} \geq 30 \sqrt{\frac{g \sin (\mu-\alpha)}{r \pi^{2} \cos (\mu+\delta)}} \quad(\mathrm{r} / \mathrm{min})
\end{aligned}
$$

Throwing movement of material particles. In addition to the forward and reverse sliding the material along the screen surface, materials may also be thrown on the screen surface. When materials take on throwing movement on the screen surface, pressure of materials onto the screen surface is $N \leq 0$, the condition for the emergence of throwing movement is as equation (13). Equation (13) can be also written as equation (14).

$$
\begin{aligned}
& -r \omega^{2} \sin \varphi_{\mathrm{d}} \sin \delta+m g \cos \alpha \leq 0 \\
& r \omega^{2} \sin \varphi_{\mathrm{d}} \geq \frac{g \cos \alpha}{\sin \delta}
\end{aligned}
$$

When $\sin \varphi_{\mathrm{d}}=1$, minimum inertial acceleration $a_{3}$ which makes materials present throwing movement can be calculated as equation (15), and minimum rotating speed of screen $n_{\mathrm{d} \text { min }}$ which makes materials thrown can be calculated as equation (16). In order to produce the throwing movement of materials out of the screen surface, the working speed of screen should be $n>n_{\mathrm{d} \min }$.

$$
\begin{aligned}
& a_{3} \geq \frac{g \cos \alpha}{\sin \delta} \\
& n_{\mathrm{d} \min } \geq 30 \sqrt{\frac{g \cos \alpha}{r \pi^{2} \sin \delta}} \quad(\mathrm{r} / \mathrm{min})
\end{aligned}
$$

Based on the above analysis, $a_{1}$ and $a_{2}$ have relationship not only with the inclined angle of screen surface $\alpha$ and the vibration direction angle $\delta$, but also with the static friction angle $\mu$. It illuminates that when $\alpha$ and $\delta$ are constant, the values of $a_{1}$ and $a_{2}$ will be different with the changing of friction angle of different materials, as shown in Table 1. As known from table 1, for the pieces of plastic film $a_{1}<a_{2}$, they are easy to slide forward along the screen surface; for the sandstones $a_{1}>a_{2}$, they are easy to reverse slide. Pieces of plastic film and sandstones move just the reverse direction, which is in line with requirements of sorting.

Table 1 Values of $a_{1}$ and $a_{2}$ of different materials on the screen surface

\begin{tabular}{c|c|c|c|c|c|c|c}
\hline \multicolumn{4}{c|}{ Pieces of plastic film } & \multicolumn{4}{c}{ Sandstones } \\
\hline \multicolumn{2}{c}{$\mu=54^{\circ}$ (Pieces of plastic film and screen mesh) } & \multicolumn{4}{c}{$\mu=32^{\circ}$ (Sandstones and screen mesh) } \\
\hline$\alpha\left(^{\circ}\right)$ & $\delta\left({ }^{\circ}\right)$ & $a_{1}\left(\mathrm{~m} / \mathrm{s}^{2}\right)$ & $a_{2}\left(\mathrm{~m} / \mathrm{s}^{2}\right)$ & $\alpha\left(^{\circ}\right)$ & $\delta\left(^{\circ}\right)$ & $a_{1}\left(\mathrm{~m} / \mathrm{s}^{2}\right)$ & $a_{2}\left(\mathrm{~m} / \mathrm{s}^{2}\right)$ \\
\hline 27 & 20 & 11.68 & 16.14 & 27 & 20 & 8.59 & 1.39 \\
25 & 25 & 11.0 & 24.90 & 25 & 25 & 8.28 & 2.19 \\
20 & 30 & 10.31 & 52.43 & 20 & 30 & 7.73 & 4.34 \\
\hline
\end{tabular}

\section{Motion Analysis on Pieces of Plastic Film and Sandstones on Counter Flow Screen}

Motion analysis on pieces of plastic film on counter flow screen. The sorting process for solid wastes on counter flow screen is actually a process where pieces of plastic film, scraps of paper, and chips of wood move towards opposite direction to sandstone particles on the screen surface. When the rotating speed of counter flow screen is $n_{+\min }<n<n_{-\min }$ and the acceleration of screen machine is 
$a_{1}<a<a_{2}$, pieces of plastic film, scraps of paper, and chips of wood can only produce positive slide and move upward along the screen surface; when the rotating speed of counter flow screen is $n_{+\min }<n>n_{-\min }$ and the acceleration of screen machine is $a_{1}<a>a_{2}$, pieces of plastic film, scraps of paper, and chips of wood etc. can produce both positive and reverse slide. Because the acceleration for pieces of plastic film etc. is $a_{1}<a_{2}$, the reverse sliding distance for pieces of plastic film etc. is less than the forward sliding distance in a vibrating cycle of screen machine. Overall, pieces of plastic film etc. are still sliding forward along the screen surface. When the rotating speed of counter flow screen is $n_{\mathrm{d} \text { min }}<n<n_{\text {threshold }}$ and the acceleration of screen machine is $a_{\text {threshold }}>a>a_{3}$, pieces of plastic film exhibit throwing motion on the screen surface, and soon they fall back to the screen surface and slide along a certain direction. Since pieces of plastic film, scraps of paper, and chips of wood etc. are very loose and light, so they produce plastic collision without causing rebound. The bounce of pieces of plastic film etc. on the screen surface depends entirely on the vibration acceleration of screen machine. Hence, the movement of pieces of plastic film on the screen surface is stable regular forward bounce and slide, as shown in Fig. 4.

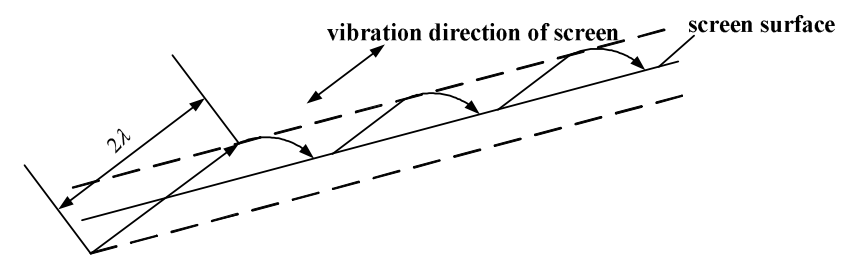

Fig. 4. Movement of pieces of plastic film on the screen surface

Motion analysis on sandstones on counter flow screen. When sandstones contact with the screen surface, they produce incomplete elastic collision to the screen surface and then bounce back. The feeding direction of machine is in accordance with the direction of normal of screen surface to some extent, so sandstones bounce back below normal and are eventually discharged from the end of the screen surface, as shown in Fig.5.

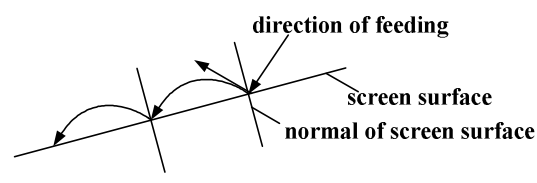

Fig. 5. Movement of sandstones on the screen surface

In general, the larger sandstone particles are, the farther to bounce back and the easier to be separated from pieces of plastic film; on the contrary, the smaller sandstone particles are, the nearer to bounce back, the easier to mix with pieces of plastic film, more difficult to be separated. When the rotating speed of counter flow screen is $n_{-\min }<n<n_{+\min }$ and the acceleration of screen machine is $a_{1}$ $<a>a_{2}$, sandstones produce only a reverse sliding on the screen surface, that is, move downwards along the screen surface; when the rotating speed of screen is $n_{+\min }<n>n_{-\min }$ and the acceleration of screen machine is, sandstones can produce both positive and reverse slide. Because the acceleration for sandstones is $a_{1}>a_{2}$, the positive sliding distance for sandstones is less than the reverse sliding distance in a vibrating cycle of screen machine. As a whole, sandstones are still sliding reversely along the screen surface. When the rotating speed of counter flow screen is $n_{\text {dmin }}<n<n_{\text {threshold, }}$, sandstones exhibit bounce on the screen surface. Because sandstones with irregular shape produce incomplete elastic collision to the screen surface, their bounce is irregular and the track is also very complicated. As sandstones' incomplete elastic collision to the screen surface result in bounce, the height they bounce on the screen surface is higher than that of pieces of plastic film. Because of the irregular shape of sandstones, the various parts are unevenly stressed and rebound direction is inconsistent when colliding. But when sandstones fall back again on the screen surface, the direction of fall is above the normal of screen surface and the direction of rebound must be below the normal of screen surface, so sandstones bounce towards the end of the screen surface. Cycle like this, the 
general bounce trend for sandstones is downwards along the screen surface. In summary, sandstones perform the reverse movement on the counter flow screen, that is, move downwards along the screen surface and are finally discharged from the end of the screen surface, which separates the pieces of plastic film from sandstones.

Sorting mechanism of counter flow screen. Sorting pieces of plastic film, scraps of paper, and chips of wood etc. from sandstones on counter flow screen is actually a process where they move towards opposite direction to sandstone particles on the screen surface. Pieces of plastic film, scraps of paper, and chips of wood etc. are very loose and light with large friction factor and small elasticity coefficient, so they produce plastic collision without causing rebound. Their movement on the screen surface depends entirely on the vibration effects of screen. Therefore, the movement of pieces of plastic film on the screen surface is stable regular forward movement. For the sandstones with small friction factor and large elastic coefficient, they produce elastic collision when contacting with the screen surface and then bounce back. Due to feeding above the normal of screen surface, so sandstones rebound towards below the normal of screen surface. When sandstones fall back again to the screen surface, their feeding direction is still above the normal of screen surface. Therefore, the rebound direction must be below the normal of screen surface, that is, sandstones exhibit the reverse movement on the screen surface. Pieces of plastic film etc. and sandstones move towards the opposite direction and are thus separated from each other.

\section{Selection on Dynamical Parameters of Counter Flow Screen}

The dynamical parameters of counter flow screen are mainly including mass of vibration $m$, frequency ratio $z_{0}$, natural frequency of main vibration $\omega_{\mathrm{n}}$, spring stiffness of main vibration $k$, spring stiffness of connecting rod $k_{0}$, required exciting force $F$, eccentricity $r$, connecting rod force $\mathrm{F}_{l}$, required power $P$, and power amplitude passed to base $F_{d}$.

(1) Mass of vibration $m$ can be calculated as equation (17).

$$
m=m_{1}+K_{m} m_{m}
$$

where, $m_{1}$ is the box weight, $\mathrm{kg} ; K_{m}$ is the combination coefficient of materials, $0.1 \sim 0.4 ; m_{m}$ is the mass of materials, $\mathrm{kg}$.

(2) Natural frequency of main vibration for spring rod counter flow screen can be calculated as following equations. Natural frequency no-load $\omega_{n k}$ and natural frequency of load $\omega_{n y}$ are calculated in equation (18) and equation (19). Frequency ratio is usually selected in the following range. Frequency ratio of no-load for linear vibration is $z_{k}=0.75 \sim 0.85$ and frequency ratio of load is $z_{y}=0.80 \sim 0.90$. The relationship between frequency ratio of no-load $z_{k}$ and frequency ratio of load $z_{y}$ is shown in equation (20).

$$
\begin{aligned}
& \omega_{n k}=\sqrt{\frac{k+k_{0}}{m_{1}}} \\
& \omega_{n y}=\sqrt{\frac{k+k_{0}}{m}} \\
& z_{k}=\omega / \omega_{n k}, \quad z_{y}=\omega / \omega_{n y}, \quad z_{k}=\sqrt{\frac{m_{1}}{m}} z_{y}
\end{aligned}
$$

(3) Resonance spring stiffness of vibration system can be obtained from equation (18) and equation (19) to equation (21). Spring stiffness of main vibration $k$ and spring stiffness of connecting $\operatorname{rod} k_{0}$ are calculated by equation (22) and equation (23).

$$
k+k_{0}=m_{1} \omega_{n k}^{2}=\frac{1}{z_{k}^{2}} m_{1} \omega^{2}=m \omega_{n y}^{2}=\frac{1}{z_{y}^{2}} m \omega^{2}
$$




$$
\begin{aligned}
& k=m \omega^{2} \\
& k_{0}=k+k_{0}-k=\left(\frac{1}{z_{y}^{2}}-1\right) m \omega^{2}
\end{aligned}
$$

(4) Required exciting force $F$ and eccentricity $r$ are achieved from equation (24) and equation $(25)$.

$$
\begin{aligned}
& F=k_{0} r=\frac{\lambda}{\cos \gamma}\left(k+k_{0}\right)\left(1-z^{2}\right)=\frac{k_{0} \lambda}{\cos \gamma} \\
& r=\frac{\lambda}{\cos \gamma}
\end{aligned}
$$

where, $\gamma=\arctan \frac{2 b z_{y}}{1-z_{y}} ; \gamma$ is the phase angle; $\lambda$ is the amplitude of counter flow screen; $b$ is the damping ratio, 0.05 0.07.

(5) Forces on the connecting rod and torque of shaft in normal working conditions are respectively calculated in equation (26) and equation (27); Forces on the connecting rod and torque of shaft when starting are respectively displayed in equation (28) and equation (29). Thereinto, $k_{0 j}$ and $k_{j}$ are respectively the static stiffness of main vibration spring and the static stiffness of connecting rod spring.

$$
\begin{aligned}
& F_{l z}=k_{0} \sqrt{\lambda^{2}+r^{2}-2 \lambda r \cos \gamma} \\
& M_{z}=0.5 k_{0} r\left(\sqrt{\lambda^{2}+r^{2}-2 \lambda r \cos \gamma}-\lambda \sin \gamma\right) \\
& F_{l q}=\frac{k_{0 j} k_{j} r}{k_{0 j}+k_{j}} \\
& M_{q}=\frac{1}{2} \times \frac{k_{0 j} k_{j} r^{2}}{k_{0 j}+k_{j}}
\end{aligned}
$$

(6) Power consumption under normal operation is calculated as equation (30).

$$
P_{z}=\frac{1}{2000 \eta} c \lambda^{2} \omega^{2} \quad(\mathrm{~kW})
$$

where, $\eta$ is the transmission efficiency, $0.90 \sim 0.95 ; \lambda$ is the amplitude of screen, $\mathrm{m} ; c$ is damping coefficient, $\mathrm{N} \cdot \mathrm{s} / \mathrm{m} ; \omega$ is the circular frequency of vibration, $1 / \mathrm{s}$.

When choosing motors for counter flow screen, power consumption at starting should be considered to select motors. Power consumption $P_{q}$ when starting can be calculated by equation (31).

$$
P_{q}=\frac{M_{q} \omega}{1000 \eta K_{c}} \quad(\mathrm{~kW})
$$

where, $K_{c}$ is the starting torque coefficient, namely the ratio of starting torque to normal torque.

(7) Power amplitude of vertical direction $F_{d c}$ and power amplitude of horizontal direction $F_{d s}$ are obtained from equation (32). Dynamic load amplitude of synthesis passed to base $F_{d}$ is calculated as equation (33).

$$
\begin{aligned}
& F_{d c}=k \lambda \sin (\alpha+\delta) \\
& F_{d s}=k \lambda \cos (\alpha+\delta) \\
& F_{d}=\sqrt{F_{d c}^{2}+F_{d s}^{2}}
\end{aligned}
$$




\section{Conclusions}

(1) Counter flow screen is a kind of new friction sorting equipment, which can be used in the sorting process for solid wastes;

(2) As long as two kinds of materials differ in friction factors and elasticity coefficient, can they be sorted by counter flow screen;

(3) The separation effects of different materials on counter flow screen depend on not only reasonable selection on dynamical parameters of screen, but also the difference in friction factors and elasticity coefficient for materials. The more difference they have in friction factors and elasticity coefficients, the better sorting performance can be achieved;

(4) In the design of similar types of separation equipment, dynamical parameters under steady working state of machine can be calculated according to the computational formula listed in this paper.

\section{Acknowledgement}

The study is supported by "the Fundamental Research Funds for the Central Universities" (N090303001).

\section{References}

[1] B. C. Wen, S. Y. Liu, Q. He, Theory and Dynamic Design Method of Mechanical Vibration, Machinery Industry Press, Beijing, 2001.

[2] B. C. Wen, S. Y. Liu, Z. B. Chen, H. Li, Theory and Application of Mechanical Vibration, Higher Education Pres, Beijing, 2009.

[3] B. C. Wen, H. Zhang, S. Y. Liu, et. al. Theory and Techniques of Vibrating Machinery and Their Applications, Science Press, Beijing, 2010.

[4] B. C. Wen, Y. N. Li, Y. M. Zhang, Vibration Utilization Engineering, Science Press, Beijing, 2005.

[5] S. Y. Liu, Y. Yuan, B. C. Wen, The Analysis of Motions of Two Types of Materials on Linear Vibrating Screen, Journal of Northeastern University(Natural Science), 18(1997) 23-27.

[6] L. Xin, B. C. Wen, Dynamic Analysis of a Vibrating Conveyer in Consideration of Materials Friction, $1^{\text {st }}$ International Conference on Vibration Utilization and Control $4^{\text {th }}$ National Congress of Vibration Utilization Engineering, 19-21, August, 2010, Shenyang, Liaoning, China.

[7] B. C. Wen, H. Li, et. Al. Friction characteristics between two bodies under the vibration working condition and their applications. Progresses in fracture and strength of materials and structures, Hainan Island, China, 353(2007) 758-761.

[8] S Y Liu, Y Yuan, B C Wen. Dynamic Analysis of a Vibration Conveyer with Large Inclination in Consideration of Material Forces. Journal of Northeastern University(Natural Science), 17(1996) 315 319

[9] Y Fang, M L Zhang, J C Wei. The Research and Application of Garbage Screening Equipment. Science Information, 2007(11): p24 25 